\title{
CONCERNING CELLULAR DECOMPOSITIONS OF 3-MANIFOLDS WITH BOUNDARY
}

\author{
BY \\ STEVE ARMENTROUT(')
}

1. Introduction. In [2], we proved that if $G$ is a cellular decomposition of a 3-manifold $M$ such that the associated decomposition space is a 3-manifold $N$, then $M$ and $N$ are homeomorphic. In this paper we shall establish a related result for 3-manifolds with boundary. We shall show that if $\boldsymbol{G}$ is a cellular decomposition of a 3-manifold with boundary $M$ such that the associated decomposition space is a 3-manifold with boundary $N$, then $M$ and $N$ are homeomorphic.

The techniques of this paper have applications to the study of embeddings of curves and surfaces in 3-manifolds. Suppose that $M$ is a 3-manifold with boundary and $G$ is a cellular decomposition of $M$ such that the associated decomposition space is a 3-manifold with boundary $N$. By Theorem 2 of this paper, $M$ and $N$ are homeomorphic. Suppose $K$ is a surface or a curve in $M$ such that no nondegenerate element of $G$ intersects $K$. If $P$ denotes the projection map from $M$ onto $N$, then $P \mid K$ is a homeomorphism. It is natural to ask the following: Is $P[K]$ embedded in $N$ the same way that $K$ is embedded in $M$ ? In $\S 5$, we give an affirmative answer to this question. In particular, $K$ is tame if and only if $P[K]$ is tame.

In $\$ 6$ we shall show that if $G$ is a cellular decomposition of a 3-manifold with boundary into a 3-manifold with boundary, then the projection map can be approximated arbitrarily closely by homeomorphisms.

In [2], we established a theorem of basic importance in the study of cellular decompositions of 3-manifolds that yield 3-manifolds as their decomposition spaces. The main result, Theorem 1, of this paper is a useful corollary of the results of [2]. The results mentioned in the preceding two paragraphs are applications of Theorem 1. In [4], we shall apply Theorem 1 to the study of shrinkability conditions which are satisfied by certain cellular decompositions of $E^{3}$ that yield $E^{3}$ as their decomposition space.

2. Terminology and notation. The statement that $M$ is a 3-manifold with boundary means that $M$ is a separable metric space such that each point of $M$ has a neighborhood in $M$ which is a 3-cell. If $M$ is a 3-manifold with boundary, a point $p$ of $M$ is an interior point of $M$ if and only if $p$ has an open neighborhood in $M$ which is an open 3-cell. The interior of $M$, Int $M$, is the set of all boundary points,

Received by the editors January 25, 1968.

(1) This research was supported in part by the National Science Foundation under Grant No. GP-3857. 
and the boundary of $M, \mathrm{Bd} M$, is $M$-Int $M$. The statement that $M$ is a 3-manifold means that $M$ is a 3-manifold with boundary such that $\mathrm{Bd} M$ is void.

If $M$ is a 3-manifold with boundary, a set $X$ in $M$ is a cellular subset of $M$ if and only if there exists a sequence $C_{1}, C_{2}, C_{3}, \ldots$ of 3-cells in $M$ such that (1) for each positive integer $n, C_{n+1} \subset \operatorname{Int} C_{n}$, and (2) $X=\bigcap_{i=1}^{\infty} C_{i}$. Note that a cellular subset of a 3-manifold with boundary $M$ necessarily lies in the interior of $M$.

If $M$ is a 3-manifold with boundary, the statement that $G$ is a cellular decomposition of $M$ means that $G$ is an upper semicontinuous decomposition of $M$ such that each element of $G$ is a cellular subset of $M$.

If $X$ is a topological space and $G$ is an upper semicontinuous decomposition of $X$, then $X / G$ denotes the associated decomposition space, $P$ denotes the projection map from $X$ onto $X / G$, and $H_{G}$ denotes the union of all the nondegenerate elements of $G$.

If $A$ is a set in a topological space $X$, let $\beta A$ denote the (topological) boundary of $A$, and let $\mathrm{Cl} A$ denote the closure of $A$. If $X$ is a metric space, $A \subset X$, and $\varepsilon$ is a positive number, then $V(\varepsilon, A)$ denotes the open $\varepsilon$-neighborhood of $A$.

3. The main result. The purpose of this section is to establish the main result of the paper. We shall depend heavily on the following result from [2].

THEOREM 1 OF [2]. Suppose that $M$ is a 3-manifold and $G$ is a cellular decomposition of $M$ such that $M / G$ is a 3-manifold $N$. Suppose that $N$ has a triangulation $T$ such that if $\sigma$ is a simplex of $T, P^{-1}[\sigma]$ lies in an open 3-cell $U_{\sigma}$ in $M$. Then there exists a triangulation $\Sigma$ of $M$ and an isomorphism $\phi$ from $T$ onto $\Sigma$ such that for each simplex $\sigma$ of $T, \phi(\sigma) \subset U_{\sigma}$.

Theorem 1. Suppose $M$ is a 3-manifold with boundary and $G$ is a cellular decomposition of $M$ such that $M / G$ is a 3-manifold with boundary $N$. Suppose $U$ is an open set in Int $N$ such that $\beta U$ misses $P\left[H_{G}\right]$. Then there is a homeomorphism hrom $\mathrm{Cl} P^{-1}[U]$ onto $\mathrm{Cl} U$ such that $h\left|\beta P^{-1}[U]=P\right| \beta P^{-1}[U]$.

Proof. We shall apply Theorem 1 of [2], and we have some preliminary steps to take before we can apply Theorem 1 of [2].

For each positive integer $k$, let $A_{k}$ be the union of all the sers of $G$ lying in $P^{-1}[U]$ and of diameter at least $1 / k$. Then by upper semicontinuity of $G, A_{k}$ is closed. It follows that there exists a sequence $V_{1}, V_{2}, V_{3}, \ldots$ of open sets in $M$ such that for each positive integer $i, V_{i}$ contains $\beta P^{-1}[U], \bar{V}_{i+1} \subset V_{i}, \bar{V}_{i}$ and $A_{i}$ are disjoint, and $V_{i} \subset V\left(1 / i, \beta P^{-1}[U]\right)$. There is an open covering $\mathscr{W}$ of $P^{-1}[U]$ such that (1) if $W \in \mathscr{W}, W$ is an open 3-cell and $\bar{W} \subset P^{-1}[U],(2)$ if $n$ is any positive integer, $W$ is a set of $\mathscr{W}$, and $W$ intersects $\bar{V}_{n+1}$, then (diam $W$ ) $<1 / n$, and (3) if $g \in G$ and $g \subset P^{-1}[U]$, then there is a set $W$ of $\mathscr{W}$ such that $g \subset W$. Such a set $\mathscr{W}$ may be constructed in the following way. If $g \in G$ and $g \subset A_{1}$, there is an open 3-cell $W_{g}$ such that $g \subset W_{g}, \bar{W}_{g} \subset P^{-1}[U]$, and $W_{g}$ and $\bar{V}_{1}$ are disjoint. If $g \in G$ and $g \subset A_{2}-A_{1}$, there is an open 3-cell $W_{g}$ such that $g \subset W_{g}, \bar{W}_{g} \subset P^{-1}[U]$, (diam $\left.W_{g}\right)<1$, and $W_{g}$ and $\bar{V}_{2}$ are disjoint. If $n$ is any positive integer, $n>1, g \in G$, and $g \subset A_{n}-A_{n-1}$, 
there is an open 3-cell $W_{g}$ such that $g \subset W_{g}, \bar{W}_{g} \subset P^{-1}[U]$, (diam $\left.W_{g}\right)<1 /(n-1)$, and $W_{g}$ and $\bar{V}_{n}$ are disjoint. Let $\mathscr{W}$ be the collection of all such sets $W_{g}$ for the elements $g$ of $G$ lying in $P^{-1}[U]$.

We shall show that if $n$ is any positive integer, $W$ is a set of $\mathscr{W}$, and $W$ intersects $\bar{V}_{n+1}$, then (diam $W$ ) $<1 / n$. If $W$ intersects $\bar{V}_{n+1}$ and $g$ is any set of $G$ lying in $A_{n+1}$, then $W$ is distinct from $W_{g}$. It follows that (diam $W_{g}$ ) $<1 / n$. The remaining properties of $\mathscr{W}$ are evident.

There is a triangulation $T$ of $U$ such that (1) if $n$ is a positive integer, $\sigma \in T$, and $\sigma$ intersects $P\left[V_{n}\right]$, then $(\operatorname{diam} \sigma)<1 / n$, and (2) if $\sigma \in T$, then $P^{-1}[\sigma]$ lies in some set of $\mathscr{W}$. For each simplex $\sigma$ of $T$, let $W_{\sigma}$ be some open 3-cell of $\mathscr{W}$ such that $P^{-1}[\sigma]$ $\subset W_{\sigma}$.

Let $G_{0}$ be the set of all elements of $G$ contained in $P^{-1}[U]$. Then $G_{0}$ is a cellular decomposition of the 3-manifold $P^{-1}[U]$. By Theorem 1 of [2], there exist a triangulation $\Sigma$ of $P^{-1}[U]$ and an isomorphism $\phi$ from $T$ onto $\Sigma$ such that if $\sigma \in T$, $\phi(\sigma) \subset W_{\sigma}$.

Since $\phi^{-1}$ is an isomorphism from $\Sigma$ onto $T$, by the proof of Lemma 8 of [2], there is a homeomorphism $h_{0}$ from $P^{-1}[U]$ onto $U$ such that if $\sigma$ is a simplex of $\Sigma$, then $h_{0}[\sigma]=\phi^{-1}(\sigma)$. Define a function $h$ as follows: (1) If $x \in P^{-1}[U]$, then $h(x)$ $=h_{0}(x)$. (2) If $x \in \beta P^{-1}[U]$, then $h(x)=P(x)$. Since $P^{-1}[\beta U]=\beta P^{-1}[U]$, and $\beta P^{-1}[U]$ and $P^{-1}[U]$ are disjoint, $h$ is well defined. Clearly $h$ is from $\mathrm{Cl}^{-1}[U]$, and since $h_{0}$ is onto $U$ and $P \mid \beta P^{-1}[U]$ is onto $\beta U, h$ is onto $\mathrm{Cl} U$. By definition of $h$, $h\left|\beta P^{-1}[U]=P\right| \beta P^{-1}[U]$. In order to complete the proof of Theorem 1 , we need only to show that $h$ is a homeomorphism.

Since $\beta U$ misses $P\left[H_{G}\right], P$ is one-to-one on $\beta P^{-1}[U]$. Since $h_{0}$ is one-to-one, it follows that $h$ is one-to-one.

Now we shall prove that $h$ is continuous. Clearly, it is sufficient to show that if $x_{1}, x_{2}, x_{3}, \ldots$ is a sequence of points in $P^{-1}[U]$ and converging to the point $x_{0}$ of $\beta P^{-1}[U]$, then $h\left(x_{1}\right), h\left(x_{2}\right), h\left(x_{3}\right), \ldots$ converges to $h\left(x_{0}\right)$, or, in view of the definition of $h$, to $P\left(x_{0}\right)$.

For each positive integer $i$, let $\tau_{i}$ be a 3-simplex of $\Sigma$ containing $x_{i}$, and let $\sigma_{i}$ be $\phi^{-1}\left(\tau_{i}\right)$. By construction of $h, h\left(x_{i}\right) \in \sigma_{i}$. Now $x_{1}, x_{2}, x_{3}, \ldots$ converges to $x_{0}$ and $x_{0} \in \beta P^{-1}[U]$. Further, for each positive integer $i, \tau_{i} \subset W_{\sigma_{i}}$. It follows from these facts and properties of $\mathscr{W}$ that (diam $\left.\tau_{1}\right),\left(\operatorname{diam} \tau_{2}\right),\left(\operatorname{diam} \tau_{3}\right), \ldots$ converges to 0 .

Let $Q$ be a neighborhood of $h\left(x_{0}\right)$. Since $h\left(x_{0}\right)=P\left(x_{0}\right)$ and $\left\{x_{0}\right\}$ is an element of $G$, $P^{-1}[Q]$ is a neighborhood of $x_{0}$. From facts mentioned in the preceding paragraph, it follows that there is a positive integer $s$ such that if $n>s, \tau_{n} \subset P^{-1}[Q]$. It follows from facts about the construction of $\mathscr{W}$ that there is a positive integer $t$ greater than $s$ such that if $n>t, W_{\sigma_{n}} \subset P^{-1}[Q]$. Hence if $n>t, P\left[W_{\sigma_{n}}\right] \subset Q$. The open covering $\mathscr{W}$ has the property that if $\sigma \in T, P^{-1}[\sigma] \subset W_{\sigma}$. Hence for each positive integer $n$, $P^{-1}\left[\sigma_{n}\right] \subset W_{\sigma_{n}}$, and if $n>t$, then both $\sigma_{n} \subset P\left[W_{\sigma_{n}}\right] \subset Q$ and $h\left(x_{n}\right) \in Q$. It follows that $h\left(x_{1}\right), h\left(x_{2}\right), h\left(x_{3}\right), \ldots$ converges to $h\left(x_{0}\right)$. Consequently $h$ is continuous.

Now we shall prove that $h^{-1}$ is continuous. It is sufficient to show that if 
$y_{1}, y_{2}, y_{3}, \ldots$ is a sequence of points in $U$ converging to the point $y_{0}$ of $\beta U$, then $h^{-1}\left(y_{1}\right), h^{-1}\left(y_{2}\right), h^{-1}\left(y_{3}\right), \ldots$ converges to $h^{-1}\left(y_{0}\right)$, or equivalently, to $P^{-1}\left[y_{0}\right]$.

For each positive integer $i$, let $\sigma_{i}$ be a 3-simplex of $T$ containing $y_{i}$, and let $\tau_{i}$ be $\phi\left(\sigma_{i}\right)$. By construction of $h$, for each $i, h^{-1}\left(y_{i}\right) \in \tau_{i}$.

Suppose $R$ is a neighborhood of $P^{-1}\left[y_{0}\right]$. Since $\left\{P^{-1}\left[y_{0}\right]\right\}$ is an element of $G$, there is a neighborhood $R_{0}$ of $P^{-1}\left[y_{0}\right]$ such that $R_{0} \subset R, R_{0}$ is a union of elements of $G$, and if $W \in \mathscr{W}$ and $W$ intersects $R_{0}$, then $W \subset R$. Notice that $P\left[R_{0}\right]$ is a neighborhood in $N$ of $y_{0}$.

There is a positive integer $t$ such that if $n>t, y_{n} \in P\left[R_{0}\right]$. If $n>t, P^{-1}\left[y_{n}\right] \in R_{0}$. Since for each $i, P^{-1}\left[\sigma_{i}\right] \subset W_{\sigma_{i}}$ and $y_{i} \in \sigma_{i}$, then if $i>t, W_{\sigma_{i}}$ intersects $R_{0}$ and hence lies in $R$. By construction of $\Sigma$, it follows that for each $i, \tau_{i} \subset W_{\sigma_{i}}$, and hence if $i>t$, $\tau_{i} \subset R$ and therefore $h^{-1}\left(y_{i}\right) \in R$. It follows that $h^{-1}\left(y_{1}\right), h^{-1}\left(y_{2}\right), h^{-1}\left(y_{3}\right), \ldots$ converges to $P^{-1}\left[y_{0}\right]$, or to $h^{-1}\left(y_{0}\right)$. Hence $h^{-1}$ is continuous.

Therefore $h$ is a homeomorphism from $\mathrm{Cl}^{-1}[U]$ onto $\mathrm{Cl} U$ such that $h \mid \beta P^{-1}[U]$ $=P \mid \beta P^{-1}[U]$. This establishes Theorem 1 .

4. Application to 3-manifolds with boundary. We are now prepared to extend Theorem 2 of [2] to the case of cellular decompositions of 3-manifolds with boundary.

THEOREM 2. Suppose $M$ is a 3-manifold with boundary and $G$ is a cellular decomposition of $M$ such that $M / G$ is a 3-manifold with boundary $N$. Then there is a homeomorphism $h$ from $M$ onto $N$ such that $h|\mathrm{Bd} M=P| \mathrm{Bd} M$.

Proof. Int $N$ is an oper subset of $N$ lying in Int $N$, and $\beta($ Int $N)=\operatorname{Bd} N$. Further, $P^{-1}[\operatorname{Int} N]=\operatorname{Int} M$, and $P^{-1}[\beta(\operatorname{Int} N)]=\mathrm{Bd} M$. We also have that $\mathrm{Cl}$ Int $M=M$ and $\mathrm{Cl}$ Int $N=N$. With the aid of Theorem 1, it follows that there exists a homeomorphism $h$ from $M$ onto $N$ such that $h|\operatorname{Bd} M=P| \operatorname{Bd} M$.

5. Applications to embeddings. In this section we establish some results concerning embeddings of surfaces and curves in manifolds. Our first result is a slightly more general theorem.

THEOREM 3. Suppose that $M$ is a 3-manifold with boundary and $G$ is a cellular decomposition of $M$ such that $M / G$ is a 3-manifold with boundary $N$. Suppose that $K$ is a closed nowhere dense subset of $M$ such that $K$ and $H_{G}$ are disjoint. Then there is a homeomorphism $h$ from $M$ onto $N$ such that $h|K=P| K$.

Proof. This follows by applying Theorem 1 to the open subset (Int $M)-K$ of $M$. Since $K$ is nowhere dense in $M, \mathrm{Cl}[(\operatorname{Int} M)-K]=M$, and it follows that there exists a homeomorphism $h$ from $M$ onto $N$ such that $h|(K \cup \operatorname{Bd} M)=P|(K \cup \operatorname{Bd} M)$. In particular, $h|K=P| K$.

COROLlary 1. Suppose that $M$ is a 3-manifold with boundary and $G$ is a cellular decomposition of $M$ such that $M / G$ is a 3-manifold with boundary $N$. Suppose $K$ is a 
manifold with boundary, of dimension 1 or 2 , contained in $M$, and missing $H_{G}$. Then there is a homeomorphism $h$ from $M$ onto $N$ such that $h|K=P| K$.

The next two corollaries may be regarded as extensions of Theorem 1 of [1].

Corollary 2. Suppose that $M$ is a 3-manifold with boundary and $G$ is a cellular decomposition of $M$ such that $M / G$ is a 3-manifold with boundary. Suppose $K$ is a 2-manifold with boundary in $M$ such that $K$ misses $H_{G}$. Then $P[K]$ is tame in $N$ if and only if $K$ is tame in $M$.

A compact connected set $X$ in $E^{3}$ is pointlike in $E^{3}$ if and only if $E^{3}-X$ is homeomorphic to $E^{3}-\{0\}$. It is well known (see [6]) that in $E^{3}$, "pointlike" and "cellular" are equivalent. By a pointlike decomposition of $E^{3}$ is meant an upper semicontinuous decomposition of $E^{3}$ into pointlike compact connected sets.

Corollary 3. Suppose that $G$ is a pointlike decomposition of $E^{3}$ such that $E^{3} / G$ is homeomorphic to $E^{3}$. Suppose $K$ is a 2-manifold in $E^{3}$ such that $K$ misses $H_{G}$. Then $P[K]$ is tame if and only if $K$ is tame.

COROLlaRY 4. Suppose that $G$ is a pointlike decomposition of $E^{3}$ such that $E^{3} / G$ is homeomorphic to $E^{3}$. Suppose that $J$ is an arc or a simple closed curve in $E^{3}$ such that $J$ misses $H_{G}$. Then $P[J]$ is tame if and only if $J$ is tame.

It follows from results of [3] and [5] that under the hypothesis of Corollary 4, if $J$ is a simple closed curve, $\pi_{1}\left(E^{3}-J\right)$ and $\pi_{1}\left(E^{3}-P[J]\right)$ are isomorphic. Corollary 1 gives a considerably stronger result in this case.

6. Approximating the projection map. The result of this section shows that if $G$ is a cellular decomposition of a 3-manifold with boundary into a 3-manifold with boundary, then the projection map can be approximated arbitrarily closely by homeomorphisms. D. R. McMillan, Jr. raised the question as to whether such approximations are possible.

THEOREM 4. Suppose that $M$ is a 3-manifold with boundary and $G$ is a cellular decomposition of $M$ such that $M / G$ is a 3-manifold with boundary $N$. Suppose $U$ is an open set in Int $M$ containing $H_{G}$ and $\varepsilon$ is a positive number. Then there exists a homeomorphism $h$ from $M$ onto $N$ such that (1) if $x \in M-U, h(x)=P(x)$ and (2) if $x \in M$, $d(h(x), P(x))<\varepsilon$.

Proof. The proof of this theorem is a modification of the proof of Theorem 1 above. Let $\mathscr{A}$ be an open covering of $N$ by sets of diameter less than $\varepsilon / 2$. Let $V_{1}, V_{2}, V_{3}, \ldots$ be as in the proof of Theorem 1 . There is an open covering $\mathscr{W}$ of $U$ such that (1) if $W \in \mathscr{W}, W$ is an open 3-cell and $\bar{W} \subset U,(2)$ if $n$ is any positive integer, $W \in \mathscr{W}$, and $W$ intersects $\bar{V}_{n+1}$, then $(\operatorname{diam} W)<1 / n$, (3) if $g \in G$ and $g \subset P^{-1}[U]$, then $g$ lies in some set of $\mathscr{W}$, and (4) if $W \in \mathscr{W}$, there is a set $A$ of $\mathscr{A}$ such that $P[W] \subset A$. 
Since $H_{G} \subset U, P[U]$ is open in $N$. There is a triangulation $T$ of $P[U]$ such that (1) if $n$ is any positive integer, $\sigma \in T$, and $\sigma$ intersects $P\left[V_{n}\right]$, then $(\operatorname{diam} \sigma)<1 / n$, and (2) if $\sigma \in T$, then $P^{-1}[\sigma]$ lies in some set of $\mathscr{W}$. For each simplex $\sigma$ of $T$, let $W_{\sigma}$ be some open 3-cell of $\mathscr{W}$ such that $P^{-1}[\sigma] \subset W_{\sigma}$.

Let $G_{0}$ be the set of all elements of $G$ contained in $U$. Then $G_{0}$ is a cellular decomposition of the 3-manifold $U$. By Theorem 1 of [2], there exist a triangulation $\Sigma$ of $U$ and an isomorphism $\phi$ from $T$ onto $\Sigma$ such that if $\sigma \in T, \phi(\sigma) \subset W_{\sigma}$. By the proof of Lemma 8 of [2], there is a homeomorphism $h_{0}$ from $U$ onto $P[U]$ such that if $\sigma \in \Sigma, h_{0}[\sigma]=\phi^{-1}(\sigma)$. Define a function $h$ as follows: (1) If $x \in M-U, h(x)=P(x)$. (2) If $x \in U, h(x)=h_{0}(x)$. As in the proof of Theorem $\mathrm{l}$, we may show that $h$ is a homeomorphism from $M$ onto $N$. By definition, if $x \in M-U, h(x)=P(x)$.

We shall show now that if $x \in M, d(h(x), P(x))<\varepsilon$. If $x \in M-U$, clearly $d(h(x), P(x))<\varepsilon$. Suppose $x \in U$. Let $\sigma$ be a 3-simplex of $T$ containing $P(x)$, and let $\tau$ be a 3-simplex of $T$ containing $h(x)$. We shall prove that $P\left[W_{\sigma}\right]$ and $P\left[W_{\tau}\right]$ intersect. First, since $P(x) \in \sigma, x \in P^{-1}[\sigma]$, and since $P^{-1}[\sigma] \subset W_{\sigma}$, then $x \in W_{\sigma}$. Second, since $h(x) \in \tau$, then by the way $h$ is defined, $x \in \phi(\tau)$. Since $\phi(\tau) \subset W_{\tau}$, then $x \in W_{\tau}$. Hence $x \in W_{\sigma} \cap W_{\imath}$, and thus $P\left[W_{\sigma}\right]$ and $P\left[W_{\tau}\right]$ intersect.

By construction of $\mathscr{W}$, if $W \in \mathscr{W}$, then for some set $A$ of $\mathscr{A}, P[W] \subset A$ and so $(\operatorname{diam} P[W])<\varepsilon / 2$. Since $x \in W_{\jmath}$, then $P(x) \in P\left[W_{\sigma}\right]$. Since $P^{-1}[\tau] \subset W_{\tau}$, then $\tau \subset P\left[W_{\imath}\right]$; since $h(x) \in \tau, h(x) \in P\left[W_{\imath}\right]$. It follows that $d(h(x), P(x))<\varepsilon$. This completes the proof of Theorem 4 .

\title{
REFERENCES
}

1. S. Armentrout, Upper semicontinuous decompositions of $E^{3}$ with at most countably many nondegenerate elements, Ann. of Math. 78 (1963), 605-618.

2. - Cellular decompositions of 3-manifolds that yield 3-manifolds, (to appear).

3. - Homotopy properties of decomposition spaces, (to appear).

4. - Shrinkability of certain decompositions of $E^{3}$ that yield $E^{3}$, Illinois J. Math. (to appear).

5. S. Armentrout and T. M. Price, Decompositions into compact sets with UV properties, (to appear).

6. D. G. Stewart, Cellular subsets of the 3-sphere, Trans. Amer. Math. Soc. 114 (1965), 10-22.

\author{
UNIVERSITY OF IOWA, \\ IOWA City, IOWA \\ UNIVERSITY OF WISCONSIN, \\ MADISON, WisCONSIN
}

